\title{
Automatic Analysis of Smoothing TECHNIQUES BY SIMULATION MODEL BASED REAL-Time System For Processing 3D Human FACES
}

\author{
Suranjan Ganguly ${ }^{1}$, Debotosh Bhattacharjee ${ }^{2}$ and Mita Nasipuri ${ }^{3}$ \\ Department of Computer Science and Engineering, Jadavpur University, India
}

\begin{abstract}
The pivotal research work that has been carried out and described in this literature acknowledges the importance of various smoothing techniques for processing 3D human faces from $2.5 \mathrm{D}$ range face images. The smoothing techniques have been developed and implemented using MATLAB-Simulink for real time processing in embedded system. In addition, the significance of smoothed $2.5 D$ range image over original face range image has been discovered as well as its time complexity has also been reported with array of experiments. The variations in time complexities are also accomplished using different optimization levels and execution modes. A set of filtering techniques such as, Max filter, Min filter, Median filter, Mean filter, Mid-point filter and Gaussian filter, have been designed and illustrated using Simulink model. The model takes depth face image (i.e. the range face image) as input in real time and presents the improvement over original face images. In the design flow, the performance of every block has also been characterized by range face images from Frav3D, GavabDB, and Bosphorus databases. In the experimental section of this research article, an array of performance analysis for these smoothing techniques with variation of frameworks is explained.
\end{abstract}

\section{KEYWORDS}

3D face image, 2.5D face image, MATLAB-Simulink, Smoothing techniques, Range face image

\section{INTRODUCTION}

Computer vision based different methodologies like object recognition, registration, identification, etc. deploys the 2D or 3D face images into automation system. Hence, the growth of image scope, and variation of applications require the computation of a complex image processing methodologies. But, sometimes these algorithms lack behind due to the presence of noise, outliers, spikes, holes, etc. For this reason, some important image data is suppressed, or lost, or some noisy data get itself processed that leads to poor performance of the particular mechanisms.

The images may incorporate variations of noises due to acquisition problems, quantization or digitization error or scanning error, etc. Now, it is very much required to filter out these noises and smooth the facial surface of the input face images for practical use of the algorithm in real time applications. In this context, the development and implementation of different linear as well as non-linear filtering techniques [1] namely: Max filter, Min filter, Mid-point filter, Mean filter, 
Gaussian filter, and Median filter have been applied on 3D human face images and it will be advantageous for further processing.

Human face images are considered to be more reliable biometric feature for automatic security system for crucial properties such as uniqueness, universality, well accepted and well understandable by people. It is always visible, and every one must have a face whereas other biometric features like hand geometry, ear, eye may be lost due to some reason. The surveillance cameras are also used to capture the human faces. Hence, face recognition [2-4] got most of the researcher's attention from last two decades.

In addition, there is a vast influence to prefer 3D human face [4] images rather than 2D images. Specifically, the 2D images preserve the reflectance characteristics of the object in the pixel data. So, it is mainly dependent on the illumination variations whereas 3D face images are particularly used to preserve the depth values in X-Y plane. Another property that makes 3D face images more convenient than $2 \mathrm{D}$ is $3 \mathrm{D}$ geometrical rotation along $\mathrm{X}, \mathrm{Y}$, and $\mathrm{Z}$ axes. Thus, the pose variation, the major problem of current face recognition, can be resolved using face registration [5-6] mechanism.

However, the states of the art of filtering techniques in case of 3D face processing reason have been summarized in table 1. In this literature study, its importance in face registration and (or) recognition has particularly an impact for developing an array of smoothing techniques implementing in real time system and illustrating their significance for processing purpose.

Table 1. The state-of-the-art of image smoothing techniques for 3D face images.

\begin{tabular}{|c|c|}
\hline Reference & Description \\
\hline$[7]$ & $\begin{array}{c}\text { Authors have demonstrated the effect of the median filter for removing sharp } \\
\text { spikes, and again interpolation technique has been added to fill the holes on the } \\
\text { face image. }\end{array}$ \\
\hline$[8]$ & $\begin{array}{c}\text { Authors have compared the performance of landmark localization technique with } \\
\text { array of smoothing methods, namely Max Filter, Min filter, Gaussian filter, Mean } \\
\text { filter, and Weighted median filter. }\end{array}$ \\
\hline$[9]$ & $\begin{array}{c}\text { Here, authors have used median and Gaussian filter for smoothing purpose. The } \\
\text { median filter is used for spikes from 3D faces and again, Gaussian filtering is } \\
\text { applied for removing surface noise. }\end{array}$ \\
\hline$[10]$ & $\begin{array}{c}\text { To detect the nose-tip, authors have computed Gradient Weighting Filter method } \\
\text { during the smoothing process of their proposed algorithm. }\end{array}$ \\
\hline
\end{tabular}

\section{Motivation AND APPLiCATION}

Studying the recent state of the art regarding the influence of smoothing techniques for 3D human face processing, authors have proposed an approach to real time processing of some of the filtering techniques using MATLAB-Simulink model.

\subsection{Range Image creation}

The 2.5D range [11] face images are gray like face images. The difference between gray $2 \mathrm{D}$ and $2.5 \mathrm{D}$ is that, $2.5 \mathrm{D}$ images are comprised by depth values (or Z's values) from $3 \mathrm{D}$ images where as $2 \mathrm{D}$ images are intensity values. Thus, the background has minimum depth value i.e. zero (0) and nose region (especially 'pronasal') landmark has a maximum depth [6] [12] value 255. In figure 
1, 2D, 2.5D and 3D face images of randomly selected subjects from Frav3D database [13] is described.

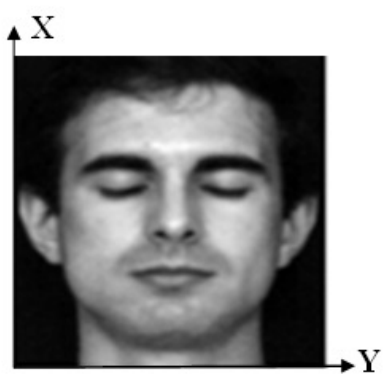

2D Face image

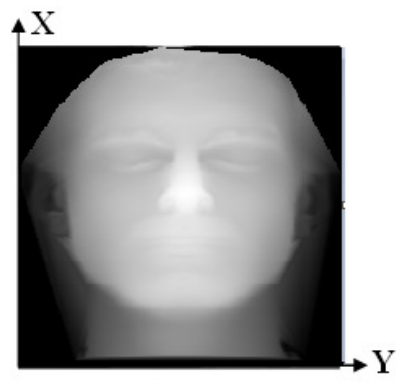

2.5D Face image

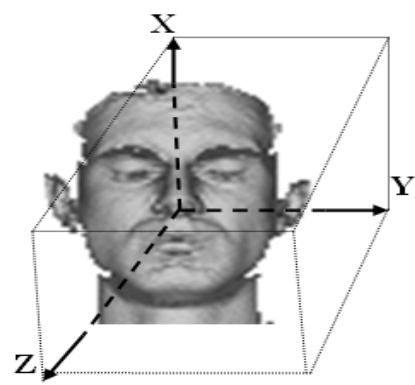

3D Face image

Figure 1. 2D, 2.5D and 3D face images of Frav3D database

Other than Frav3D face database, GavabDB [14] and Bosphorus [15] face databases have also been considered for emphasizing the significance of smoothing technique using a simulation model [19-20] of the embedded system. In figure 2, created range face images of randomly selected subject from GavabDB and Bosphorus database have been illustrated in figure 2.

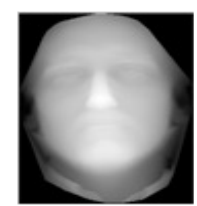

(a) From GavabDB database

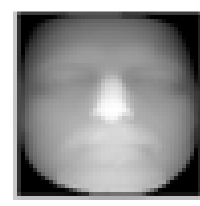

(b) From Bosphorus database

Figure 2. Created range face image

\subsection{Smoothing algorithms}

During the investigation phase, authors have implemented spatial linear as well as order-statistic [1] [16] (i.e. non linear) filters on depth values of $2.5 \mathrm{D}$ range face images. The linear filters [17] specifically an Mean filter and Gaussian filter are computed whereas in order statistic categorization of image filtering, Median filter, Max filter, Min filter, and Midpoint filter are applied on range face images.

\subsubsection{Preprocessing technique}

Before, these series of filters are experimented on depth values for their significance, a preprocessing task have been carried out. The range images have been padded by zeros in the opposite side of each row and column of the image. Thus, each and every depth values from the furthest row and column of the image can be processed for better performance analysis. Otherwise, it would not be considered during spatial image processing purpose. This phenomenon is shown in figure 3 . In this figure, a block of depth values with $8 \times 8$ grid from a section of $2.5 \mathrm{D}$ range face image is shown. 


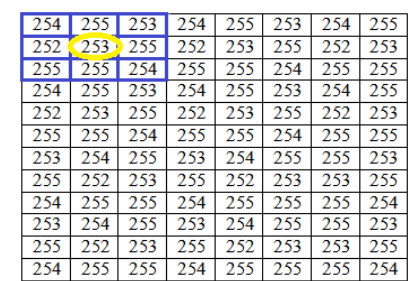

(a) without padding

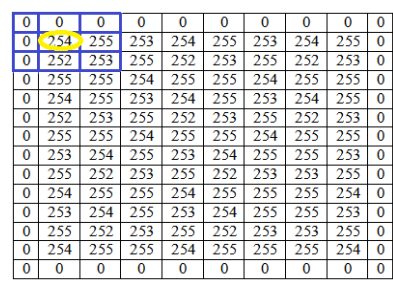

(b) with zero padding

Figure 3. The importance of depth image padding

The highlighted (circled by yellow color) depth value is processed first by the smoothing techniques. If the smoothing technique is used on original range image then, the far most two rows and columns will be unchanged, whereas with padding zeros with the original image will effectively affect these sections. This padding is done in real time and again it has been removed after the filtering techniques have been applied. Thus, the dimension is preserved before and after smoothing technique.

\subsubsection{Smoothing by linear filter}

Linear filters do not depend on any kind of order of depth values (or intensity) from filtering kernel. The filters in this category only compute the linear functions (like Gaussian or Averaging) for removing the noises irrespective of the ordering of values encompassed by the filtering window.

Gaussian filter: It is an important filter among set of smoothing filters from linear class. The weight of the Gaussian filter [1] [16] is chosen from the Gaussian kernel. For the qualitative measurement during this research work for 2.5D depth face image, 2D Gaussian kernel is implemented. The kernel function [16] with $\sigma=3$ is computed. It is observed that, a large value of $\sigma$ i.e. variance has the wider filter and smoothing impact.

Mean filter: Mean filter [1] is simple linear spatial filter that averages the neighbor's depth values of the filter mask. It is also referred as low pass filter [18]. To analyze the effect of the averaging or mean filter for depth face images, a $3 \times 3$ kernel have been undertaken.

\subsubsection{Smoothing by nonlinear filter}

These filters are also known as order-statistic filter [1]. It is a nonlinear smoothing filter whose output is emphasized on the ordering of the values encompassed by the filtering mask. Now, the output from the ranking result is used to modify the center depth value of the mask. Here, for nonlinear order statistic filter, authors have also considered for computed $3 \times 3$ kernel filter mask.

Max filter: In this noise filtering mechanism, $100 \%$ or highest depth value from neighborhood depth values is chosen. Hence, for depth based image filtering scheme, the holes (containing minimum or ' 0 ' depth value) may be removed.

Min filter: It is useful to select the minimum or $0 \%$ depth value among the selected data by the filtering window. Hence, the spikes (containing maximum depth) within the human face surface due to scanning error can be minimized.

Mid-point filter: It is another type of smoothing technique which is used to select the depth value in between maximum and minimum. It is a similar type of Mean filter as described above. 
Median filter: It is one of the famous and well known order-statistic filtering scheme where, $50 \%$ among 9-values selected by $3 \times 3$ filtering window. It has another qualitative property that, it provides us less blurring effect [1] than linear filters.

The different outputs from these filters have been demonstrated in the discussion section where the significance of each output is broadly discussed.

\subsection{Discussion}

In this section, the outcomes of respective filters of randomly selected subjects from three databases are shown in figure 4.

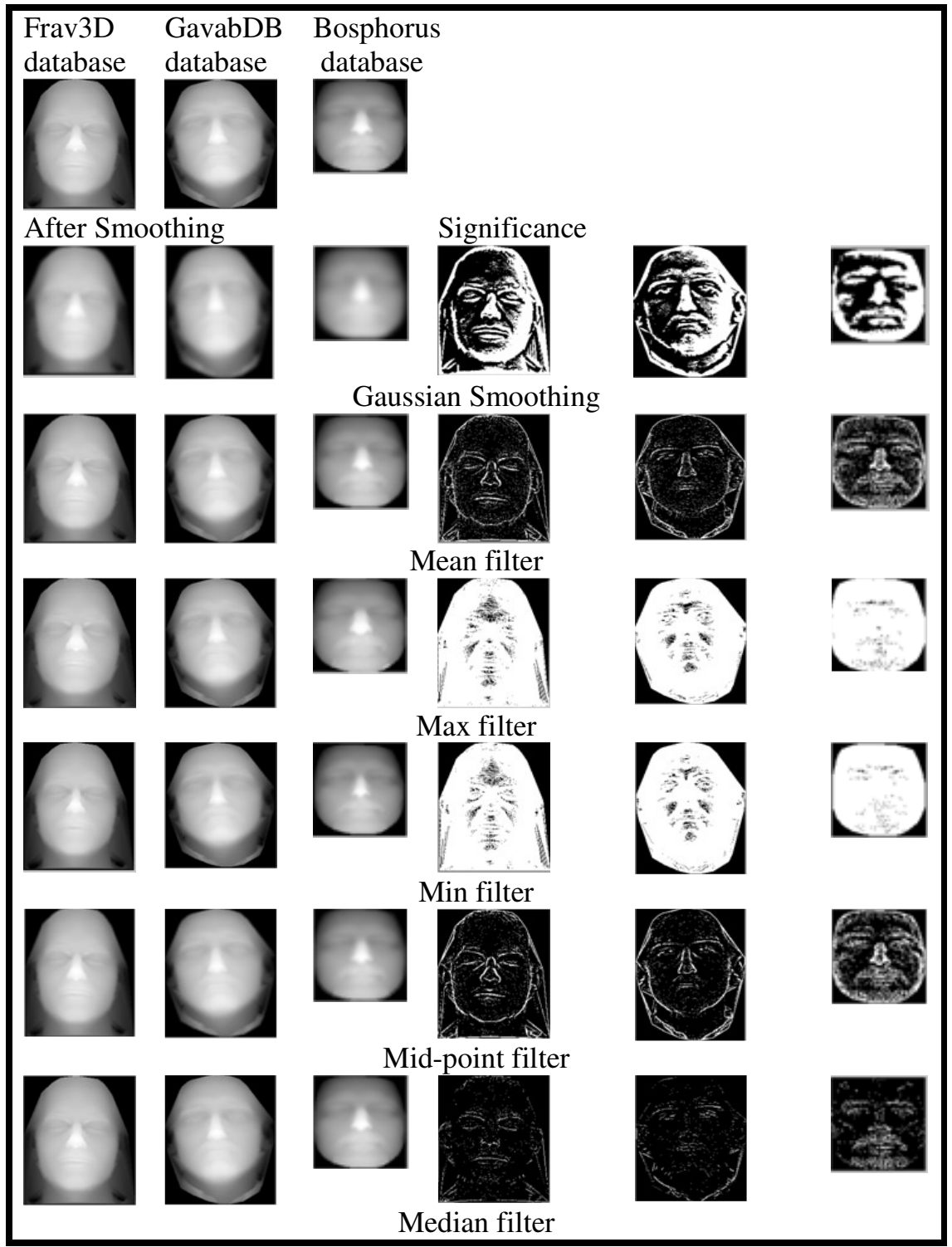

Figure 4. Visualization of smoothing effects 
In this observation, it is noticed that the smoothing technique has a great significance over depth values of the human face. So, its application in real time may significantly improve different aspects of processing human face like face registration, recognition, etc.

Now, from Gaussian analysis it is noted that the outer portion is blurred out more than other. It actually shrinks to center as it is dense and simultaneously blurs the edges. Hence, depth values near different facial regions like eyes, eyebrows, the nose region, and lips all have this quality. It is the property of the Gaussian filter, and it has successfully been executed in real time for depth values. In the case of another linear filter namely, Mean filter the edges are preserved. In these points, the depth value is nearly same as the average value that has been computed by $3 \times 3$ window. In might have a greater significance for landmark localization, face component extraction, etc. The same significance has also been found for Mid-point filtering technique. By mathematical logic it is determining $50 \%$ i.e. in between maximum and minimum, likely same as average filter. For Max and Min filter, authors have observed the same significance after smoothing technique. The reason of such significant output is that it is either selecting $0 \%$ or $100 \%$ depth value under the filtering window. Thus, it almost has a binary thresholded image as shown in [11]. Hence, the spikes and holes can be removed in this process. The most well known order statistic Median filtering method preserves the elliptical concave and convex curve details near eye region, the nose region, lip region, etc.

\section{MODEL DESIGN AND IMPLEMENTATION}

Model has been designed and implemented using MATLAB-Simulink environment. Different modules from Simulation tool have been coupled to finalize the implemented model. The detailing of the blocks has been illustrated later in this section. It is an approach for real time human computer interaction for visualizing the significant effect of different filtering techniques on 3D human faces. Not only model design, successful code-generation has also been attempted. In figure 5, developed model has been described.

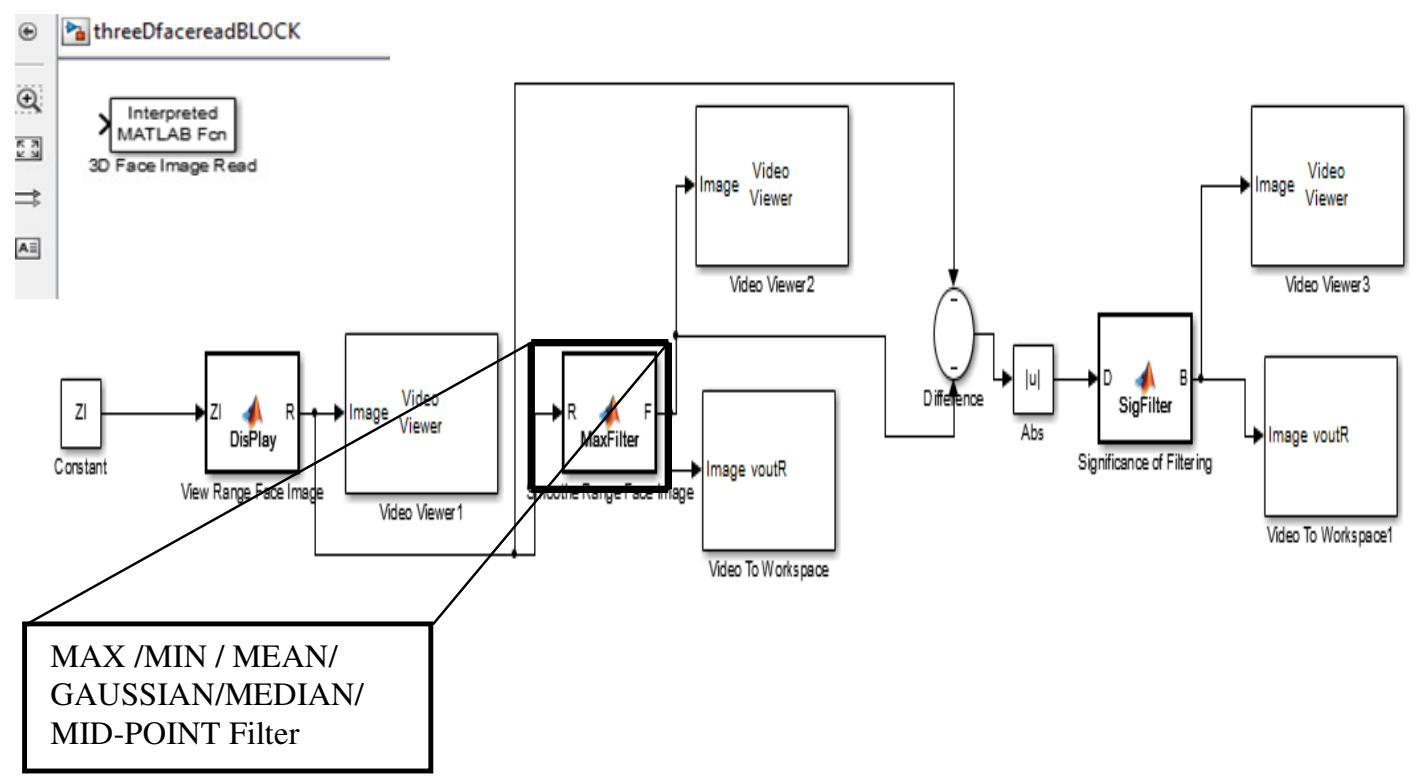

Figure 5. Illustration of developed models 
In the figure, the zoomed region shows different user defined embedded MATLAB function blocks that have been designed for real time processing in embedded system.

In general, three 'User-Defined MATLAB Function Block', four 'Sinks' blocks, one 'Constant Source', two 'Math Operations' block are incorporated with the advanced model. Each of these blocks has its own crucial role for successful implementation of an advanced model.

The 3D face images [11] are far more different from 2D images as described earlier in the motivation section. Instead of intensity values, depth (values along Z-axis) in X-Y plane is preserved in 3D images in '.wrl', '.abs,' '.bnt' like formats. Hence, before the smoothing technique is applied on 2.5D range face image, it has been generated from 3D face image using an 'Interpreted MATLAB Function' (shown in upper-left corner of figure 5). The 'Constant Source' is used as input of depth values to the Simulation model. Now, 'MATLAB Function Blocks' are allowed to embed the source code for displaying the range face image and then it is processed, and significance has been highlighted. The 'Sinks' have been used to produce an output from each block for better human computer interaction. Finally, two mathematical operations are used for real time 2D matrix manipulation purpose.

After, the successful implementation of 'Simulation' model, it is further required to generate code for embedded system. For this purpose successful code generation have been accomplished by choosing 'C-language' as target language with 'Optimization on' parameter of Compiler optimization level, 'Fixed-step' solver option (i.e. fixed step size of 0.02) along with 'Auto generated comments'. In figure 6, code generation report for Mid-point filter is shown.

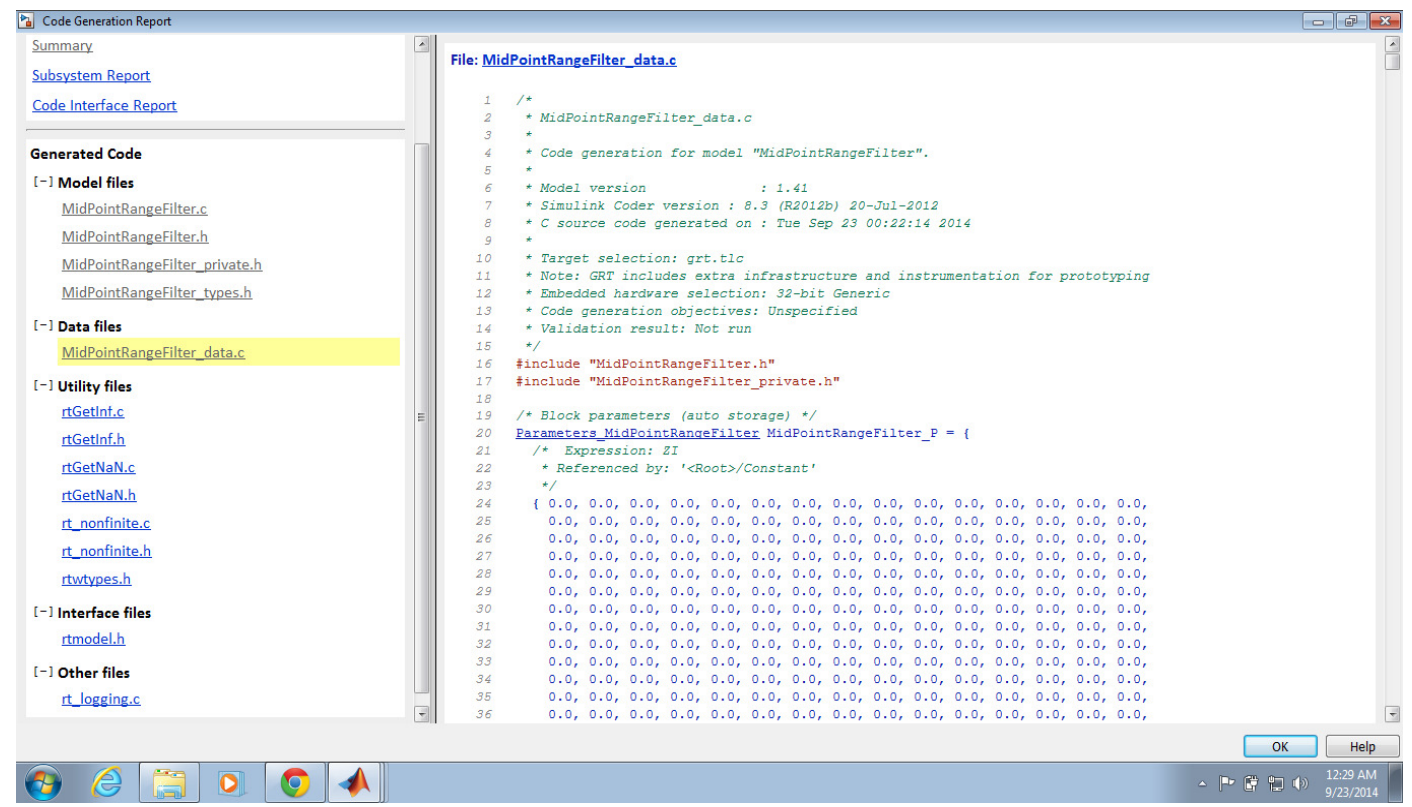

Figure 6. Description of the code generation report

In this figure, the expression of ' $\mathrm{ZI}$ ', the constant block from the model, is highlighted. It contains the depth values from range image which have been used during the execution time of the model. 


\section{EXPERIMENTAL RESULT}

Though there are other parameters for validating the model for real time application, but authors have used two variations among them. One is Simulation mode i.e. whether the model is tested by Normal mode or Accelerator or Rapid Accelerator mode and another parameter is Compiler Optimization level (either Optimizations off or Optimizations on).

In table 2, an array of analysis of this execution parameters with simulation stop time 1.0 is summarized. This description is used for the range image that has been selected randomly from Frav3D database. It has been tested on 4-GB RAM with Windows 7 (64-bit) professional Operating System environment and Inrel i5-3470 CPU with 3.20GHz processors.

[

Table 2. Performance analysis of different parameter configuration

\begin{tabular}{|c|c|c|c|}
\hline \multirow[t]{2}{*}{ Smoothing Techniques } & \multirow{2}{*}{$\begin{array}{l}\text { Simulation Mode } \\
\text { Simulation } \\
\text { Stop time 1.0 }\end{array}$} & \multicolumn{2}{|c|}{ Compiler Optimization Level } \\
\hline & & Optimizations off & Optimizations on \\
\hline \multirow{3}{*}{ Gaussian Filter } & Normal & 13.967028 seconds & 9.988573 seconds \\
\hline & Accelerator & $\begin{array}{c}9.907918 \text { seconds } \\
\text { [After Successfully built } \\
\text { the Accelerator target] }\end{array}$ & $\begin{array}{c}9.813664 \text { seconds } \\
\text { [After Successfully } \\
\text { built the Accelerator } \\
\text { target] }\end{array}$ \\
\hline & Rapid Accelerator & $\begin{array}{c}12.664001 \text { seconds } \\
\text { [After Successfully built } \\
\text { the rapid accelerator } \\
\text { target] }\end{array}$ & $\begin{array}{l}11.924632 \text { seconds } \\
\text { [After Successfully } \\
\text { built the rapid } \\
\text { accelerator target] }\end{array}$ \\
\hline \multirow{3}{*}{ Mean filter } & Normal & 11.504452 seconds & 6.921782 seconds \\
\hline & Accelerator & $\begin{array}{c}6.954723 \text { seconds } \\
\text { [After Successfully built } \\
\text { the Accelerator target] }\end{array}$ & $\begin{array}{c}6.503646 \text { seconds } \\
\text { [After Successfully } \\
\text { built the Accelerator } \\
\text { target] }\end{array}$ \\
\hline & Rapid Accelerator & $\begin{array}{c}9.511578 \text { seconds } \\
\text { [After Successfully built } \\
\text { the rapid accelerator } \\
\text { target] }\end{array}$ & $\begin{array}{l}9.285953 \text { seconds } \\
\text { [After Successfully } \\
\text { built the rapid } \\
\text { accelerator target] }\end{array}$ \\
\hline \multirow{3}{*}{ Max filter } & Normal & 7.275049 seconds & 7.225375 seconds \\
\hline & Accelerator & $\begin{array}{c}6.528666 \text { seconds } \\
\text { [After Successfully built } \\
\text { the Accelerator target] }\end{array}$ & $\begin{array}{c}6.706566 \text { seconds } \\
\text { [After Successfully } \\
\text { built the Accelerator } \\
\text { target] }\end{array}$ \\
\hline & Rapid Accelerator & $\begin{array}{c}10.056241 \text { seconds } \\
\text { [After Successfully built } \\
\text { the rapid accelerator } \\
\text { target] }\end{array}$ & $\begin{array}{l}9.704065 \text { seconds } \\
\text { [After Successfully } \\
\text { built the rapid } \\
\text { accelerator target] }\end{array}$ \\
\hline \multirow{5}{*}{ Min filter } & Normal & 7.599231 seconds & 7.000915 seconds \\
\hline & Accelerator & $\begin{array}{c}6.622307 \text { seconds } \\
\text { [After Successfully built } \\
\text { the Accelerator target] }\end{array}$ & $\begin{array}{c}6.610166 \text { seconds } \\
\text { [After Successfully } \\
\text { built the Accelerator } \\
\text { target] }\end{array}$ \\
\hline & Rapid Accelerator & $\begin{array}{c}9.467616 \text { seconds } \\
\text { [After Successfully built } \\
\text { the rapid accelerator } \\
\text { target] }\end{array}$ & $\begin{array}{l}10.232370 \text { seconds } \\
\text { [After Successfully } \\
\text { built the rapid } \\
\text { accelerator target] }\end{array}$ \\
\hline & Normal & 11.867022 seconds & 7.518357 seconds \\
\hline & & 7.109918 seconds & 7.186364 seconds \\
\hline
\end{tabular}


International Journal of Embedded Systems and Applications(IJESA) Vol.4,No.4,December 2014

\begin{tabular}{|c|c|c|c|}
\hline \multirow{2}{*}{ Mid-point filter } & Accelerator & $\begin{array}{c}\text { [After Successfully built } \\
\text { the Accelerator target] }\end{array}$ & $\begin{array}{c}\text { [After Successfully } \\
\text { built the Accelerator } \\
\text { target] }\end{array}$ \\
\cline { 2 - 4 } & Rapid Accelerator & $\begin{array}{c}10.006641 \text { seconds } \\
\text { [After Successfully built } \\
\text { the rapid accelerator } \\
\text { target] }\end{array}$ & $\begin{array}{c}\text { 9.296432 seconds } \\
\text { [After Successfully } \\
\text { built the rapid } \\
\text { accelerator target] }\end{array}$ \\
\hline Median filter & Normal & 12.546941 seconds & 12.620668 seconds \\
\cline { 2 - 4 } & Accelerator & $\begin{array}{c}12.267989 \text { seconds } \\
\text { [After Successfully built } \\
\text { the Accelerator target] }\end{array}$ & $\begin{array}{c}12.691126 \text { seconds } \\
\text { [After Successfully } \\
\text { built the Accelerator } \\
\text { target] }\end{array}$ \\
\cline { 2 - 4 } & Rapid Accelerator & $\begin{array}{c}10.047549 \text { seconds } \\
\text { [After Successfully built } \\
\text { the rapid accelerator } \\
\text { target] }\end{array}$ & $\begin{array}{c}\text { 9.809040 seconds } \\
\text { [After Successfully } \\
\text { built the rapid } \\
\text { accelerator target] }\end{array}$ \\
\hline
\end{tabular}

From this outline, it is noticed that the complexity is much higher for the techniques which are required mathematical computation much higher than others. The Mid-point filter takes more time than Max and (or) Min filter, whereas Median filter also accounts more time for smoothing operation in real time. It requires ordering of depth values encompassed by the filtering window. The Gaussian filter also consumes more time to process 3D human face image for real time application.

In figure 7, a comparative study is shown among the time complexities of different smoothing methods with an array of parameters arrangement.

\section{Compiler Optimization level: Optimizations off}

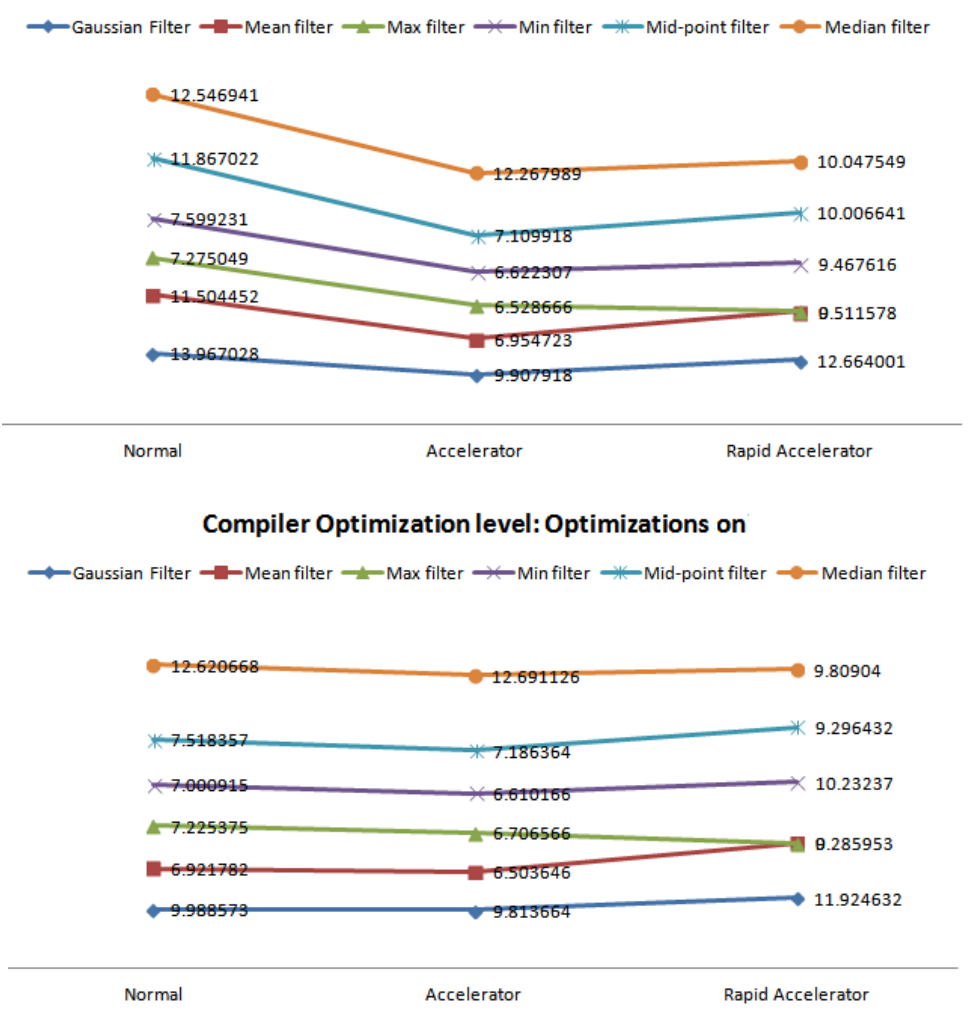

Figure 7. Comparison of the performance study 
Selecting the two restrictions such as Simulation mode and Compiler optimization level is explained here. The 'Normal', 'Accelerator' and 'Rapid Accelerator' modes are defined to compare the time complexities side by side for better understanding of the minimum time span of the specified filtering technique. Along with this, with the Compiler optimization parameter setup, fastest execution (by Optimizations on) and fastest compilation (by Optimizations off) has also been represented.

\section{CONCluSions}

The applications of smoothing techniques during image processing or computer vision (especially in face recognition) have a crucial implication. In this literature, authors have explained the influence of some of the smoothing techniques 2.5D face images. In addition, authors have created a real time application model by MATLAB-Simulink model and validated by series of parameters' composition. The code generation has also been conducted and implemented. Along with this, the validation of the model is done on three modern 3D face databases (namely Frav3D, GavabDB, and Bosphorus) having two different 3D image formats like '.wrl' and '.bnt'.

Now, authors are focusing to implement these methods for range face images using Field Propagation Gateway Array (FPGA) to develop better dedicated system with much lesser time complexity.

\section{ACKNOWLEDGEMENTS}

Authors are thankful to a project supported by DeitY (Letter No.: 12(12)/2012-ESD), MCIT, Govt. of India, at Department of Computer Science and Engineering, Jadavpur University, India for providing the necessary infrastructure for this work.

\section{REFERENCES}

[1] Gonzalez, R. C., And Woods, R.E., (2007) "Digital Image Processing," 3rd Edition, Prentice Hall Publisher.

[2] “Face Recognition,” Pp. 1-10, August 2006, Url: ttp://Www.Biometrics.Gov/Documents/Facerec.Pdf.

[3] Jelsovka, D., Hudec, R., Breznan, M., Kamencay, P., (2012) "2d-3d Face Recognition Using Shapes Of Facial Curves Based On Modified Cca Method”, 22nd International Conference Radioelektronika (Radioelektronika), Pp. 1-4.

[4] Ganguly, S., Bhattacharjee, D., And Nasipuri, M., (2014) “3d Face Recognition From Range Images Based On Curvature Analysis”, Volume: 04, Issue: 03, Pp. 748-753.

[5] Ayyagari, V.R., Boughorbel, F., Koschan, A., Abidi, M.A., (2005) "A New Method For Automatic 3d Face Registration", Proceedings Of The Ieee Computer Society Conference On Computer Vision And Pattern Recognition (Cvpr'05), Pp. 1-8.

[6] Ganguly, S., Bhattacharjee, D., And Nasipuri, M., (2014) "Range Face Image Registration Using Efri From 3d Images", In Advances In Intelligent And Soft Computing, Springer, Accepted In Proceedings Of 3rd Frontiers Of Intelligent Computing: Theory And Applications (Ficta 2014).

[7] Soltana, W. B., Ardabilian, M. Lemaire, P., Huang, D., Szeptycki, P., Chen, L., Erdogmus, N., Daniel, L., Dugelay, J., Amor, B.B., Drira, H., Daoudi, M., Colineau, J., (2012) "3d Face Recognition: A Robust Multi-Matcher Approach To Data Degradations”, In Proc Of Icb 2012, Pp 103-110.

[8] Bagchi, P., Bhattacharjee, D., Nasipuri, M., \& Basu, D.K. (2012) “A Novel Approach To Nose-Tip And Eye-Corners Detection Using H-K Curvature Analysis In Case Of 3d Images”, In Proc Of International Journal Of Computational Intelligence And Informatics, Vol. 2: No. 1.

[9] Hatem, H., Beiji, Z., Majeed, R., Lutf, M., Waleed, J., (2013) "Nose Tip Localization In ThreeDimensional Facial Mesh Data", International Journal Of Advancements In Computing Technology (Ijact), Volume5, Number13,Pp. 99-105. 
[10] Margret N. Silva, Vipul Dalal, (2013) “ Nose Tip Detection Using Gradient Weighting Filter Smoothing," International Journal Of Engineering Research And Development, Volume 9, Issue 5, Pp. 09-11.

[11] Ganguly, S., Bhattacharjee, D., And Nasipuri, M., (2014) “2.5d Face Images: Acquisition, Processing And Application", Computer Networks And Security, International Conference On Communication And Computing (Icc-2014), Organized By Alpha College Of Engineering, India, Publisher: Elsevier Science And Technology, Pp. 36-44 Isbn: 978935107244.

[12] Dhane, P., Jain, A., Kutty, K. K., (2011) "A New Algorithm For 3d Object Representation And Its Application For Human Face Verification", International Conference On Image Information Processing, Pp. 1-6.

[13] Frav3d Face Database, Url: Http://Www.Frav.Es/Databases/Frav3d/

[14] Gavabdb Face Database, Url: Http://Gavab.Escet.Urjc.Es/Recursos_En.Html

[15] Bosphorus Face Database, Url: Http://Bosphorus.Ee.Boun.Edu.Tr/Default.Aspx

[16] Jayaraman, S., Esakkirajan,S., And Veerakumar, T., (2010), "Digital Image Processing”, 3rd Edition, Tmh Publisher.

[17] Linear Filters, Url: ttp://Luthuli.Cs.Uiuc.Edu/ Daf/Courses/Cs5432009/Week\%203/Simplefilters.Pdf

[18] Spatial Filters - Mean Filter, Url: Http://Homepages.Inf.Ed.Ac.Uk/Rbf/Hipr2/Mean.Htm

[19] Ganguly, S., Bhattacharjee, D., And Nasipuri, M., (2014) "Analyzing The Performance Of HaarWavelet Transform On Thermal Facial Image Using Matlab-Simulink Model”, Proceedings Of The 1st International Conference On Microelectronics, Circuit And Systems,: Volume 2, Pages: 106-111, Isbn:81-85824-46-0.

[20] Tfrs Using Simulink, Url: Https://Www.Youtube.Com/Watch?V=31-Qd2zv5xs\&Feature=Youtu.Be

\section{AUTHORS}

SURANJAN GANGULY received the M.Tech (Computer Technology) degree from Jadavpur University, India, in 2014. He completed B-Tech (Information Technology) in 2011. His research interest includes image processing, pattern recognition. He was a project fellow of UGC, Govt. of India, sponsored major research project at Jadavpur University. Currently, he is a project fellow of DietY (Govt. of India, MCIT) funded research project at Jadavpur University.

BHATTACHARJEE received the MCSE and Ph.D. (Eng.) degrees from Jadavpur University, India, in 1997 and 2004 respectively. He was associated with different institutes in various capacities until March 2007. After that he joined his Alma Mater, Jadavpur University. His research interests pertain to the applications of computational intelligence techniques like Fuzzy logic, Artificial Neural Network, Genetic Algorithm, Rough Set Theory, etc. in Face Recognition, OCR, and Information Security. He is a life member of Indian Society for Technical Education (ISTE, New Delhi), Indian Unit for

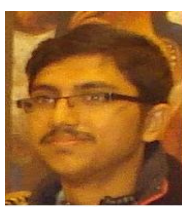
Pattern Recognition and Artificial Intelligence (IUPRAI), and a senior member of IEEE (USA).

MITA NASIPURI received her B.E.Tel.E., M.E.Tel.E., and Ph.D. (Engg.) degrees from Jadavpur University, in 1979, 1981 and 1990, respectively. Prof. Nasipuri has been a faculty member of J.U since 1987. Her current research interest includes image processing, pattern recognition, and multimedia systems. She is a senior member of the IEEE, U.S.A., Fellow of I.E. (India) and W.B.A.S.T, Kolkata, India.

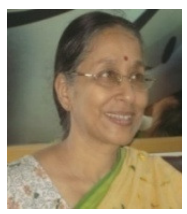

